\title{
Long period variations of dm-radio and X-ray fluxes in three X-class flares
}

\author{
H. Mészárosová ${ }^{1}$, M. Karlický ${ }^{1}$, J. Rybák ${ }^{2}$, F. Fárník ${ }^{1}$, and K. Jiřička ${ }^{1}$ \\ 1 Astronomical Institute of the Academy of Sciences of the Czech Republic, 25165 Ondřejov, Czech Republic \\ e-mail: hana@asu.cas.cz \\ 2 Astronomical Institute, Slovak Academy of Sciences, 05960 Tatranská Lomnica, Slovak Republic
}

Received 2 June 2006 / Accepted 24 August 2006

\begin{abstract}
Aims. Long period ( $\geq 60 \mathrm{~s}$ ) variations of the radio $(0.8-4.5 \mathrm{GHz})$ and X-ray fluxes observed during the July 14, 2000, April 12, 2001, and April 15, 2001 flares by the Ondřejov radiospectrograph and Yohkoh spacecraft are studied by statistical methods. Methods. In the flares under study, characteristic periods are searched for by the Fourier and wavelet methods. To understand the origin of the $0.8-4.5 \mathrm{GHz}$ drifting burst with long period variations, observed at the beginning of the April 15, 2001 flare, crosscorrelations, time shifts, coherence, and phase differences in its time series are computed.

Results. The global statistical study of these flares revealed characteristic periods in the interval of $60-513 \mathrm{~s}$ in the radio $(0.8-4.5 \mathrm{GHz})$ and $60-330 \mathrm{~s}$ in the X-ray Yohkoh fluxes. Cross-correlations between the radio fluxes at different frequencies helped us to determine the bursts generated by plasma or gyro-synchrotron mechanisms. In the April 12, 2001 flare, soft X-ray fluxes of the sources located at the loop-top and footpoints of a flare loop vary with the period of 60-320 s, and they are highly correlated. But their relation to the radio $(1.1 \mathrm{GHz}$ - plasma emission and $4.0 \mathrm{GHz}$ - gyro-synchrotron emission) is complex. At the beginning of the April 15, 2001 flare, in the $0.8-4.5 \mathrm{GHz}$ range, a broadband drifting radio burst with the time variation of $61-320 \mathrm{~s}$ was observed at times of flare loop ejection. Its detailed statistical analysis shows that this burst consists of two parts, and, that first part is generated by the plasma emission mechanism and the second, probably, by the gyro-synchrotron one. The characteristic period of about $300 \mathrm{~s}$ found in three $\mathrm{X}$-class flares in their dm-radio and X-ray emissions is discussed.
\end{abstract}

Key words. Sun: radio radiation - Sun: corona - Sun: flares - Sun: oscillations

\section{Introduction}

Oscillatory phenomena in solar flares give us important information about the flare process itself. These phenomena have been searched for in all available types of observations of solar flares in a broad range of timescales (for review see Aschwanden 2005). Most of these studies were made analyzing radio and X-ray emissions (see Tables 7.6 and 7.7 and references in Aschwanden 2005). The studied periods were usually shorter than $60 \mathrm{~s}$. Nevertheless, there are several papers that reported periods longer than $60 \mathrm{~s}$ (Aurass \& Mann 1987; Chernov et al. 1998; Gelfreikh et al. 1999; Nindos et al. 2002).

On the other hand, in TRACE observations the coronal loop oscillations with the periods in the range of 120-520 s were reported (Aschwanden et al. 1999, 2002; De Moortel et al. 2000; Nakariakov \& Ofman 2001; King et al. 2003). Nakariakov et al. (2004) numerically studied the evolution of a coronal loop in response to an impulsive energy release. They have shown that the loop density evolution curves exhibit quasi-periodic perturbations associated with the second standing harmonics of an acoustic wave.

Recently, Kislyakov et al. (2006) presented examples of variations of the solar flare microwave emission and suggested their possible connection to the photospheric 5-min oscillations. The authors discussed the role of the p-mode in such variations, and they proposed a modulation of the electric current in the coronal magnetic loop as their most probable cause. Furthermore, Nakariakov et al. (2006) proposed a new model for quasi-periodic modulation of solar flare emission, in which fast magnetoacoustic oscillations of a non-flaring loop interact with a nearby flare reconnection region.

Several types of physical mechanisms, which produce quasiperiodic phenomena in solar flares, were proposed: 1) MHD oscillations of coronal loops (Aschwanden 2005; Nakariakov \& Verwichte 2005); 2) intrinsic oscillations in the nonlinear regime of the kinetic plasma instabilities (Fleishman et al. 1994); and 3) the modulation of the acceleration process (e.g., Kliem et al. 2000). Mechanisms 2 and 3 are usually considered for processes with short periods $(\leq 1 \mathrm{~s})$. For longer periods the most probable basic cause of the periodic phenomena is some type of MHD oscillation.

In this paper we are interested in the long period $(\geq 60 \mathrm{~s})$ variations as well as in other more detailed characteristics of the radio $(0.8-4.5 \mathrm{GHz})$ and $\mathrm{X}$-ray fluxes observed during the July 14, 2000, April 12, 2001, and April 15, 2001 flares. In Sect. 2 we outline the observations. Then in Sect. 3, characteristic periods in the radio and $\mathrm{X}$-ray fluxes in the whole flare intervals are searched for. Special attention is devoted to the analysis of X-ray fluxes from the loop-top and footpoints sources of the April 12, 2001 flare. Then, the computation of crosscorrelations, time shifts, coherence, and phase differences in time series at the origin of the broadband drifting burst with long period variations, observed at the beginning of the April 15, 2001 flare, is analyzed. Discussion of results and conclusions are in Sect. 4 


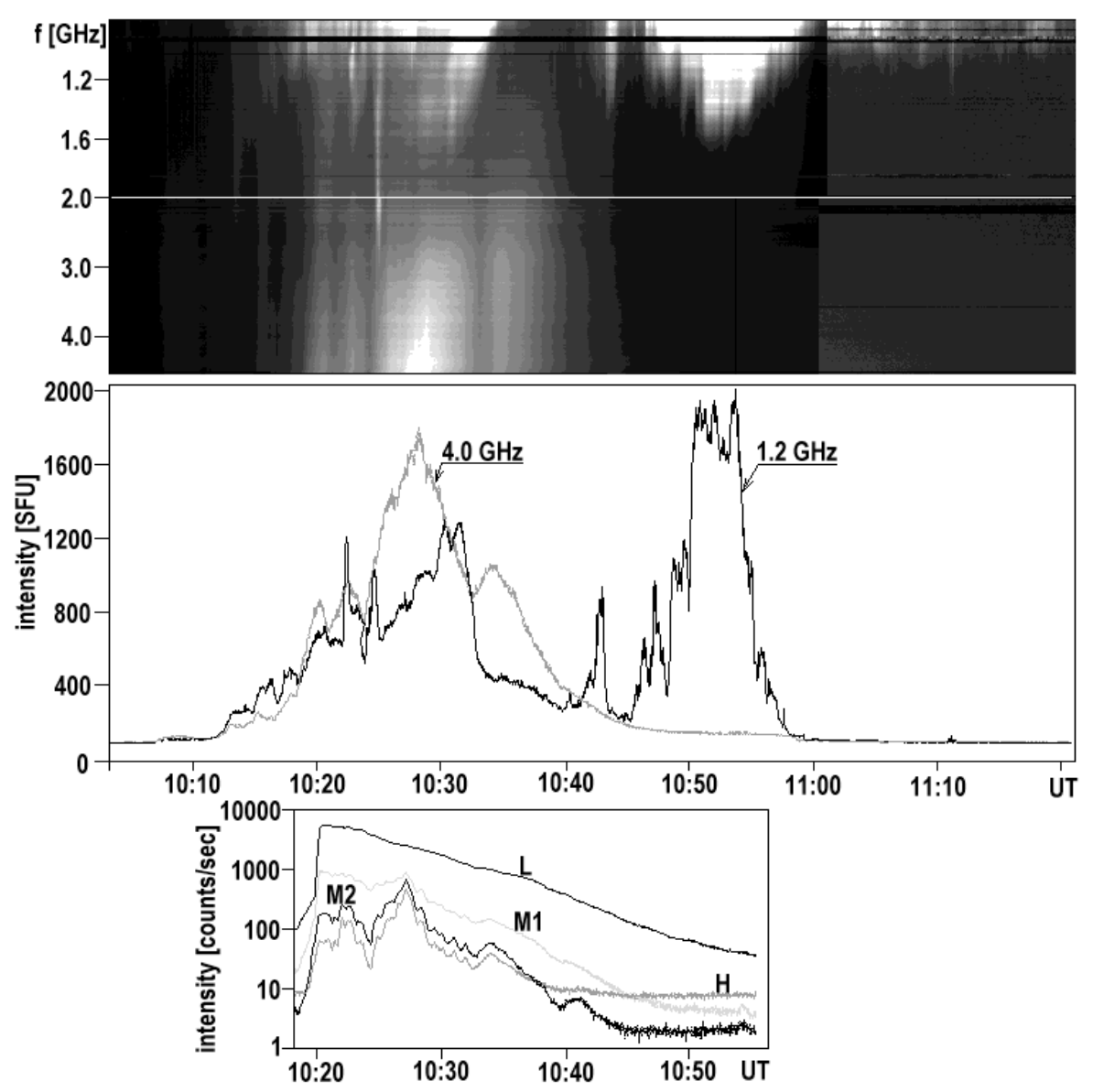

Fig. 1. The $0.8-4.5 \mathrm{GHz}$ radio spectrum (upper panel), radio fluxes (middle panel) at 1.2 and $4.0 \mathrm{GHz}$ (in grey), and Yohkoh X-ray fluxes (bottom panel: $\mathrm{L}=14-23 \mathrm{keV}, \mathrm{M} 1=23-33 \mathrm{keV}$ (in light grey), $\mathrm{M} 2=33-53 \mathrm{keV}$, and $\mathrm{H}=53-93 \mathrm{keV}$ (in grey)) of the July 14, 2000 flare. The horizontal white line (upper panel at $2 \mathrm{GHz}$ ) divides the spectra from two independent parts of the Ondřejov radiospectrograph.

Table 1. Basic characteristics of studied flares.

\begin{tabular}{|c|c|c|c|c|c|c|c|c|c|c|c|c|}
\hline \multirow[b]{2}{*}{ Flare } & \multicolumn{4}{|c|}{ GOES } & \multicolumn{6}{|c|}{$\overline{\mathrm{H}_{\alpha}}$} & \multicolumn{2}{|c|}{ Radio } \\
\hline & $\begin{array}{l}\text { Start } \\
\text { [UT] }\end{array}$ & $\begin{array}{l}\text { Max } \\
\text { [UT] }\end{array}$ & $\begin{array}{l}\text { End } \\
\text { [UT] }\end{array}$ & $\begin{array}{l}\text { X-ray } \\
\text { Imp. }\end{array}$ & $\begin{array}{l}\text { Start } \\
\text { [UT] }\end{array}$ & $\begin{array}{l}\text { Max } \\
\text { [UT] }\end{array}$ & $\begin{array}{l}\text { End } \\
\text { [UT] }\end{array}$ & $\begin{array}{l}\text { Optical } \\
\text { Imp. }\end{array}$ & Position & $\begin{array}{c}\text { NOAA } \\
\text { AR \# }\end{array}$ & $\begin{array}{l}\text { Start } \\
\text { [UT] }\end{array}$ & $\begin{array}{l}\text { End } \\
\text { [UT] }\end{array}$ \\
\hline Jul. 14, 2000 & $10: 03$ & $10: 24$ & $10: 43$ & $\mathrm{X} 5.7$ & $10: 12$ & $10: 21$ & $11: 46$ & $3 \mathrm{~B}$ & N22W07 & 9077 & $10: 03$ & $11: 31$ \\
\hline Apr. 12, 2001 & 09:39 & $10: 28$ & $10: 49$ & $\mathrm{X} 2.0$ & $10: 20$ & $10: 40$ & $11: 26$ & $2 \mathrm{~B}$ & S19W43 & 9415 & 10:13 & 11:01 \\
\hline Apr. 15, 2001 & $13: 19$ & $13: 50$ & $22: 00$ & X14.4 & $13: 36$ & $13: 49$ & $15: 35$ & $2 \mathrm{~B}$ & S20W85 & 9415 & $13: 31$ & $16: 21$ \\
\hline
\end{tabular}

\section{Observations}

For our study, three X-class flares (July 14, 2000, April 12, 2001, and April 15, 2001, see Figs. 1, 2, and 5) observed simultaneously with the $0.8-4.5 \mathrm{GHz}$ Ondřejov radiospectrograph (Jiřička et al. 1993, the time and frequency resolution is $0.1 \mathrm{~s}$ and $5 \mathrm{MHz}$, respectively) and the Yohkoh satellite (Ogawara et al. 1991) were selected. The basic characteristics of these flares are summarized in Table 1. All these flares are characterized by broadband, long-lasting radio pulsations with a broad range of characteristic timescales. In this paper we mainly focus on long period variations $(\geq 60 \mathrm{~s})$.

\subsection{The July 14, 2000 event}

According to the GOES soft X-ray observation this flare classified as X5.7 started at 10:03 UT, its maximum was at 10:24 UT, and it ended at 10:43 UT (Table 1). In $\mathrm{H} \alpha$ the flare has the importance 3B and was located in the NOAA 9077 active region. The radio spectrum of this flare in the $0.8-4.5 \mathrm{GHz}$ range together with the radio fluxes on 1.2 and $4 \mathrm{GHz}$ and the hard X-ray fluxes in four energy channels $(\mathrm{L}=14-23 \mathrm{keV}$, $\mathrm{M} 1=23-33 \mathrm{keV}, \mathrm{M} 2=33-53 \mathrm{keV}$, and $\mathrm{H}=53-93 \mathrm{keV}$ ) recorded by the HXR/Yohkoh satellite are shown in Fig. 1. The radio spectrum shows broadband pulsations in a broad range of timescales. It appears that the radio spectrum consists of two groups of broadband pulsations $(\delta f / f>0.4)$ : those from about $1.4 \mathrm{GHz}$ up to above $4.5 \mathrm{GHz}$, and those below $1.4 \mathrm{GHz}$, i.e., the frequency dividing these groups is at about $1.4 \mathrm{GHz}$. The hard X-ray emission was observed only during the rising phase of both the 1.2 and $4.0 \mathrm{GHz}$ radio emissions in the first global enhancement. The second global enhancement (10:40-11:00 UT) of the $1.2 \mathrm{GHz}$ radio flux has no counterparts in the $4.0 \mathrm{GHz}$ and $\mathrm{X}$-ray fluxes.

\subsection{The April 12, 2001 event}

This flare was classified as X2.0. In soft X-rays it started at 09:39 UT, its maximum was at 10:28 UT, and it ended at 


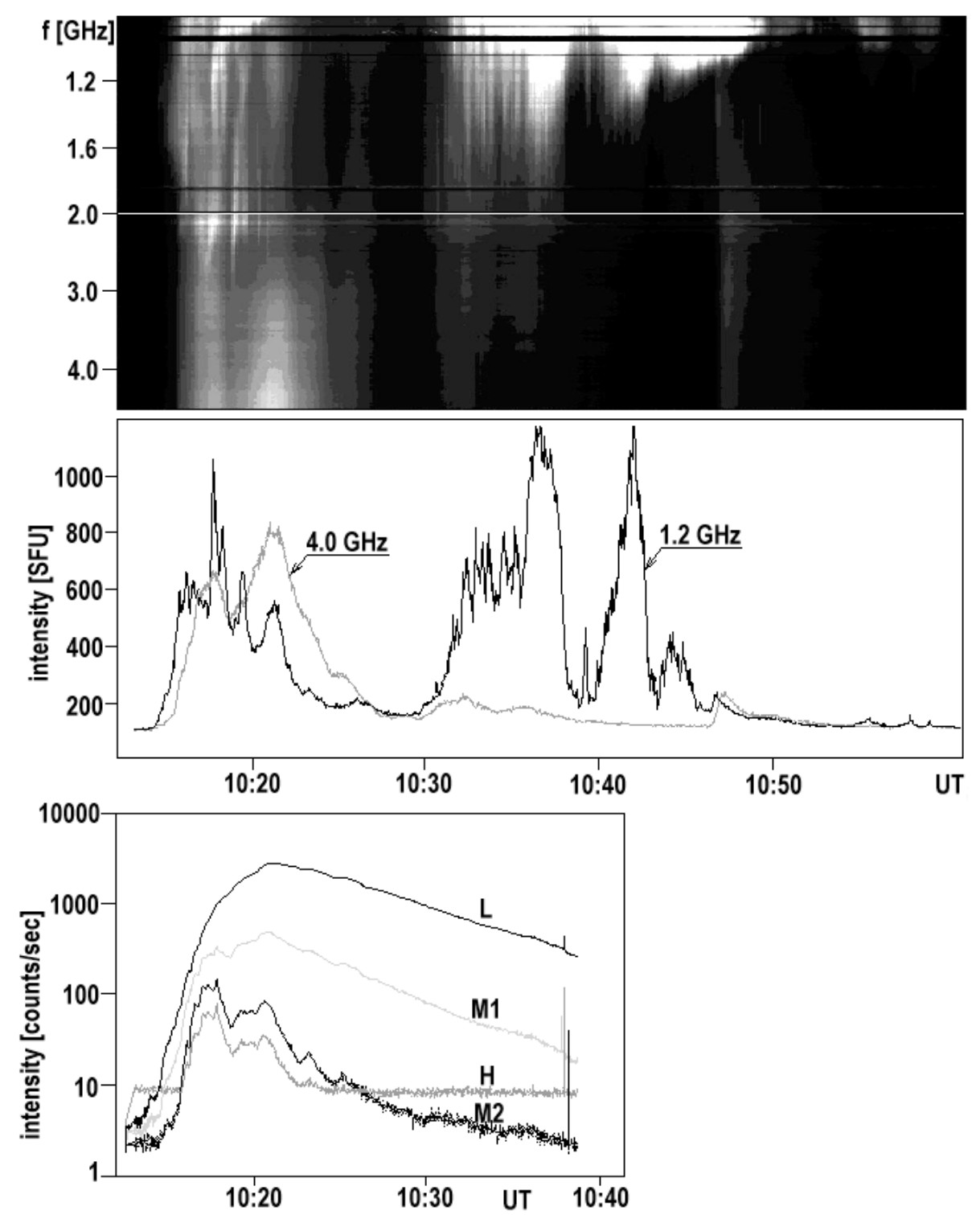

Fig. 2. The 0.8-4.5 GHz radio spectrum (upper panel), radio fluxes (middle panel) at 1.2 and $4.0 \mathrm{GHz}$ (in grey), and Yohkoh X-ray fluxes (bottom panel: $\mathrm{L}=14-23 \mathrm{keV}, \mathrm{M} 1=23-33 \mathrm{keV}$ (in light grey), $\mathrm{M} 2=33-53 \mathrm{keV}$, and $\mathrm{H}=53-93 \mathrm{keV}$ (in grey)) of the April 12, 2001 flare. The horizontal white line (upper panel at $2 \mathrm{GHz}$ ) divides the spectra into two independent parts of the Ondřejov radiospectrograph.

10:49 UT (Table 1). In H $\alpha$ the flare has the importance $2 \mathrm{~B}$ and was located in the NOAA 9415 active region. The radio spectrum of this flare in the $0.8-4.5 \mathrm{GHz}$ range together with the radio fluxes observed on 1.2 and $4.0 \mathrm{GHz}$ and the hard $\mathrm{X}$-ray fluxes recorded by the HXR/Yohkoh satellite are shown in Fig. 2. Also in this flare, the radio spectrum shows broadband pulsations in a broad range of timescales. The pulsations can be divided into two groups: those from about $1.5 \mathrm{GHz}$ up to about $4.5 \mathrm{GHz}$, and those below $1.5 \mathrm{GHz}$. On radio waves the flare started at 10:14 UT at the frequency $1.5 \mathrm{GHz}$ with the oppositely drifting features (see Fig. 3). The hard X-ray emission was recorded only during the first part of the $0.8-4.5 \mathrm{GHz}$ radio burst. Similarly to the case of the July 14, 2000 flare, in the time of the second global enhancement (10:30-10:50 UT) of the $1.2 \mathrm{GHz}$ radio flux the hard X-ray fluxes only decrease.

To understand the physical origin of the studied long period variations that are analyzed in the dm-radio and hard X-ray fluxes, we also searched for such variations in soft X-rays ( $\sim 2 \mathrm{keV}$ ) observed by the SXT/Yohkoh experiment, where even positions of the X-ray sources can be localized (Fig. 4). As seen

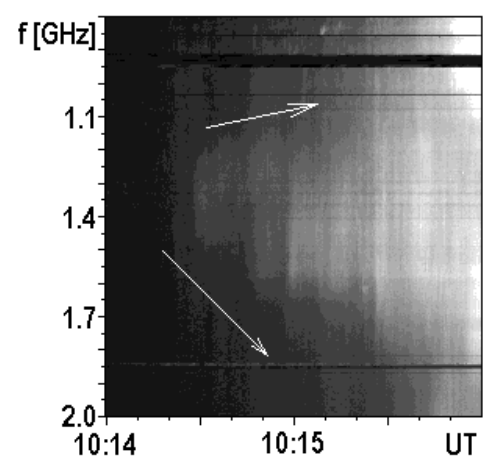

Fig. 3. Detail of the $0.8-2.0 \mathrm{GHz}$ radio spectrum of the April 12, 2001 flare. The flare started at 10:14 UT at the frequency $1.5 \mathrm{GHz}$ with the oppositely drifting features - see the arrows.

here, the X-ray emission from the loop-top source A, after the rise phase (after 10:24 UT), varies roughly with a period of about $5 \mathrm{~min}$. A detailed analysis follows. 
Table 2. Time intervals of the radio and X-ray observations studied by statistical methods (Dur. means duration).

\begin{tabular}{ccccccccccccc}
\hline \hline & \multicolumn{4}{c}{$800-2000 \mathrm{MHz}$} & \multicolumn{4}{c}{$2000-4500 \mathrm{MHz}$} & \multicolumn{3}{c}{ Yohkoh hard X-rays } & \multicolumn{3}{c}{ Yohkoh soft X-rays } \\
Flare & Start & End & Dur. & Start & End & Dur. & Start & End & Dur. & Start & End & Dur. \\
& {$[\mathrm{UT}]$} & {$[\mathrm{UT}]$} & {$[\mathrm{s}]$} & {$[\mathrm{UT}]$} & {$[\mathrm{UT}]$} & {$[\mathrm{s}]$} & {$[\mathrm{UT}]$} & {$[\mathrm{UT}]$} & {$[\mathrm{s}]$} & {$[\mathrm{UT}]$} & {$[\mathrm{UT}]$} & {$[\mathrm{s}]$} \\
\hline July 14, 2000 & $10: 03$ & $11: 22$ & 4740 & $10: 03$ & $11: 20$ & 4620 & $10: 19$ & $10: 56$ & 2204 & - & - & \\
April 12, 2001 & $10: 13$ & $11: 01$ & 2880 & $10: 13$ & $10: 57$ & 2640 & $10: 12$ & $10: 38$ & 1586 & $10: 16$ & $10: 37$ & 1260 \\
April 15, 2001 & $13: 31$ & $14: 11$ & 2400 & $13: 31$ & $14: 11$ & 2400 & $13: 37$ & $13: 55$ & 1087 & - & - & \\
\hline
\end{tabular}

Table 3. Long periods ( $\geq 60$ s) found in the July 14, 2000, April 12, 2001, and April 15, 2001 flares in the radio fluxes on 23 selected frequencies in the range $0.8-4.5 \mathrm{GHz}$.

\begin{tabular}{|c|c|c|c|c|c|c|c|c|c|}
\hline $\begin{array}{c}\text { Frequency } \\
\text { [GHz] }\end{array}$ & \multicolumn{3}{|c|}{$\begin{array}{c}\text { July } 14,2000 \\
\text { Periods } \\
\text { [s] }\end{array}$} & \multicolumn{3}{|c|}{$\begin{array}{c}\text { April 12, } 2001 \\
\text { Periods } \\
\text { [s] }\end{array}$} & \multicolumn{3}{|c|}{$\begin{array}{c}\text { April 15, } 2001 \\
\text { Periods } \\
{[\mathrm{s}]}\end{array}$} \\
\hline 0.81 & & - & & & - & & 343 & 104 & \\
\hline 0.90 & & - & & & - & & 267 & 185 & 77 \\
\hline 1.00 & & - & & & - & & 300 & 185 & 67 \\
\hline 1.02 & & - & & 320 & 160 & 76 & 300 & 185 & \\
\hline 1.10 & & - & & 288 & 160 & 87 & 300 & 185 & \\
\hline 1.20 & 339,237 & & 95 & 320 & 160 & 93 & 300 & 185 & 67 \\
\hline 1.30 & 339 & & 95 & 320 & 160 & 93,78 & 300 & 185 & \\
\hline 1.40 & 339 & & 71 & 320 & & & 300 & 185,104 & \\
\hline 1.50 & 339 & & 71 & 320 & & & 320 & 185 & 80 \\
\hline 1.60 & 474 & 116 & 71 & 320 & 160 & & 300 & 109 & 86 \\
\hline 1.70 & 279 & 116 & 71 & 288 & 160 & & 300 & 185 & \\
\hline 1.80 & 279 & & & 288 & 160 & & 300 & 185 & 86 \\
\hline 1.90 & 339 & & & & 128 & 93 & 300 & 185 & \\
\hline 2.05 & 330 & & & & 128 & 93 & & - & \\
\hline 2.25 & 330 & & & 440 & & 60 & 343 & 172 & 65 \\
\hline 2.50 & 330 & 139 & & 293 & & 98 & 267 & 185 & 61 \\
\hline 2.75 & 513,330 & 140 & 77 & 293 & 139 & 94 & 300 & 185 & 86 \\
\hline 3.00 & 513,330 & 144 & 90,78 & 293 & 139 & & 300 & 185 & 86 \\
\hline 3.25 & 513,330 & 144,125 & 80 & 293 & 139 & & 300 & 185 & 86 \\
\hline 3.50 & 513 & 144,125 & 73 & 293 & 139 & 64 & 300 & 185 & 86 \\
\hline 3.75 & 513 & 144,125 & 73 & 293 & 139 & 68 & 300 & 185 & 86 \\
\hline 4.00 & 513 & 178,115 & 60 & 293 & 115 & 64 & 300 & 185 & 86 \\
\hline 4.25 & 513 & 178,115 & 73 & 293 & 115 & 64 & 267 & 185 & 86 \\
\hline
\end{tabular}
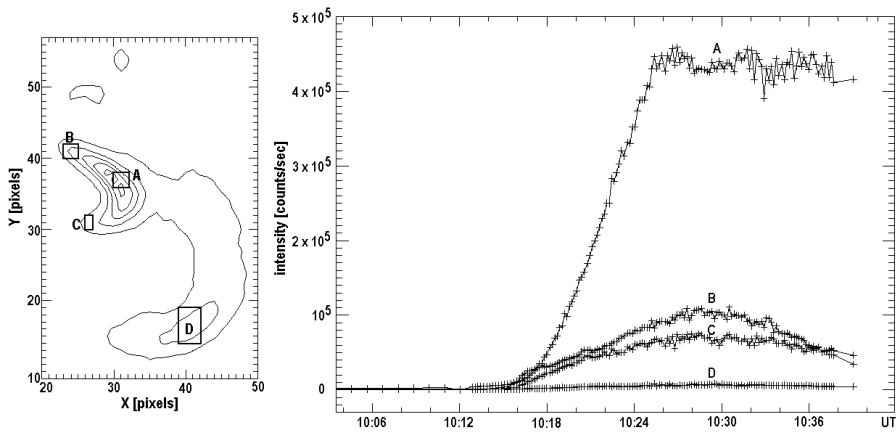

Fig. 4. Selected areas $(A=$ loop-top source, $B$ and $C=$ footpoint sources, $\mathrm{D}=$ loop-top source of the extended loop) and corresponding SXT/Yohkoh X-ray ( 2 keV) fluxes of the April 12, 2001 flare.

\subsection{The April 15, 2001 event}

This flare classified as X14.4 started at 13:19 UT, its maximum was at 13:50 UT, and it ended at 22:00 UT (Table 1). In $\mathrm{H} \alpha$ the flare has the importance $2 \mathrm{~B}$ and was located in the NOAA 9415 active region. The radio spectrum of this flare in the $0.8-4.5 \mathrm{GHz}$ range together with the radio fluxes observed at 1.2 and $4.0 \mathrm{GHz}$ and the hard X-ray fluxes recorded by the HXR/Yohkoh satellite during the first flare burst are shown in Fig. 5. The radio spectrum shows broadband pulsations in a broad range of timescales.
It is interesting that the first strong broadband burst is slowly drifting towards lower frequencies $\left(\mathrm{d} f / \mathrm{d} t \sim-21 \mathrm{MHz} \mathrm{s}^{-1}\right.$, see also Fig. 9). This burst was observed at the time of the flare loop ejection observed by the TRACE satellite (Fig. 6); see also the position of the 53-93 keV X-ray source.

\section{Statistical analysis}

First, the time intervals of the radio and X-ray observations of the three studied flares are selected for the statistical analysis (Table 2). The long period variations $\geq 60 \mathrm{~s}$ are searched for in these intervals in both radio and X-ray flux time series. Then, after the cross-correlation analysis of these time series, the unique broadband drifting burst observed at the beginning of the April 15, 2001 flare is studied.

\subsection{Global analysis: long period variations $\geq 60 \mathrm{~s}$ of radio fluxes at selected frequencies}

It is known that in the decimetric range radio spectrum there is a boundary that divides the band (characterized by fine structures and frequency drifts) with the dominant plasma emission mechanism from that (characterized by smooth continua and pulsations) where the gyro-synchrotron mechanism is dominant. To study periods in both these bands and to see their relationship we have chosen 23 frequencies (see Table 3) that 


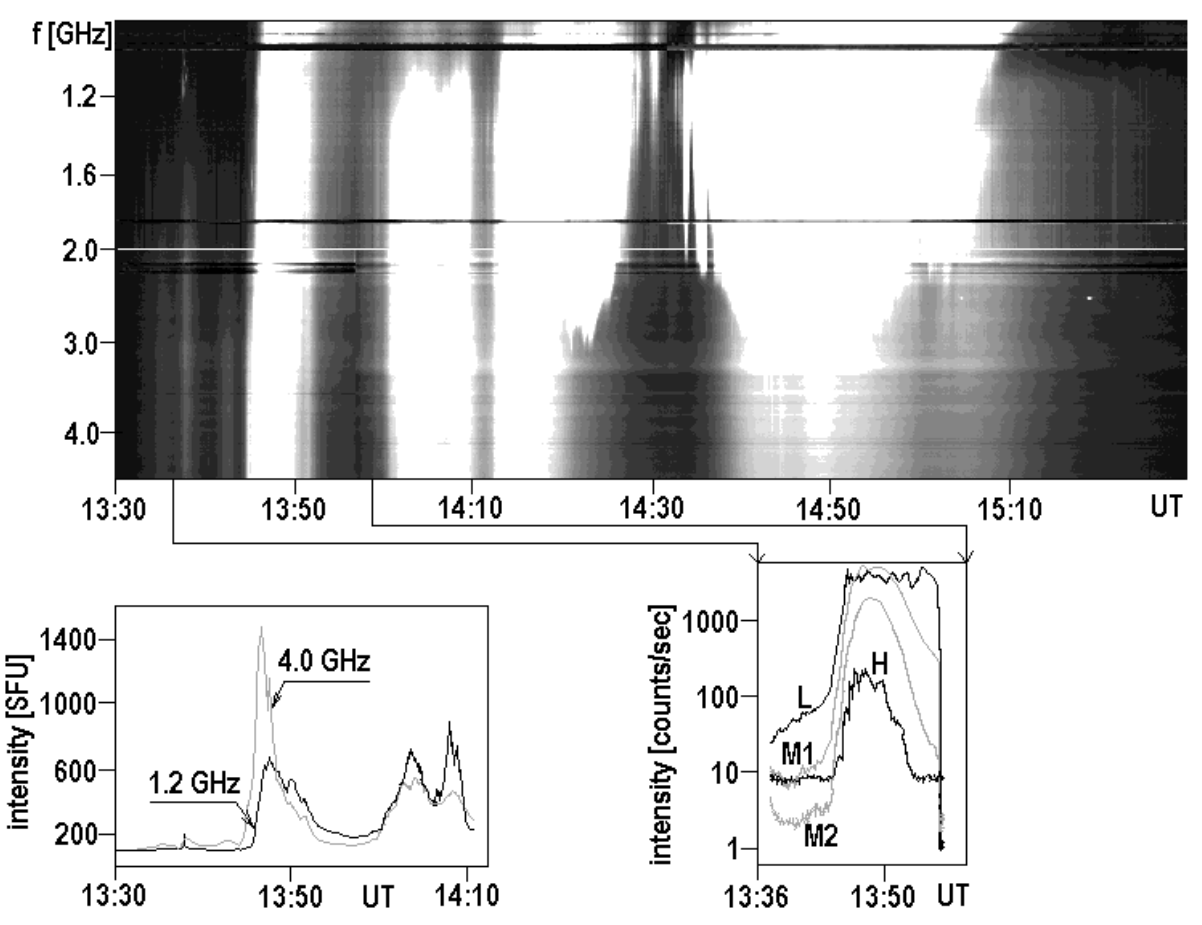

Fig. 5. The $0.8-4.5 \mathrm{GHz}$ radio spectrum (upper panel), radio fluxes (middle panel) at 1.2 and $4.0 \mathrm{GHz}$ (in grey), and Yohkoh X-ray fluxes (bottom panel: $\mathrm{L}=14-23 \mathrm{keV}, \mathrm{M} 1=23-33 \mathrm{keV}$ (in grey), $\mathrm{M} 2=33-53 \mathrm{keV}$ (in grey), and $\mathrm{H}=53-93 \mathrm{keV}$ ) of the April 15, $2001 \mathrm{flare}$. The horizontal white line (upper panel at $2 \mathrm{GHz}$ ) divides the spectra from two independent parts of the Ondřejov radiospectrograph. There is flux $\mathrm{L}$ saturation in time interval 13:45-13:55 UT.

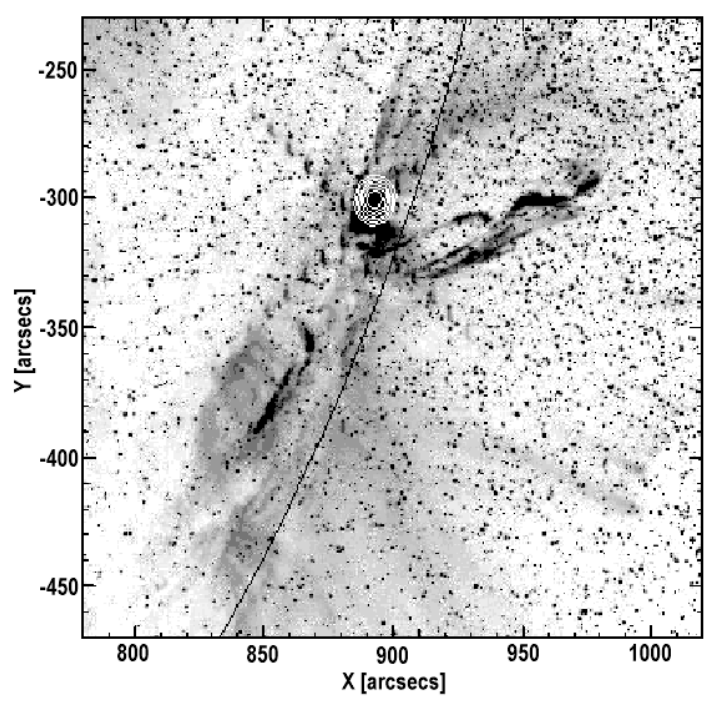

Fig. 6. The TRACE $171 \AA$ image of the loop ejection and the associated 53-93 keV hard X-ray source observed by the Yohkoh satellite (white contours) at 13:45:56 UT during the April 15, 2001 flare.

cover the whole frequency band of the Ondřejov radiospectrograph $(0.8-4.5 \mathrm{GHz})$. At all these frequencies, in the whole time intervals shown in Table 2 , the periods $\geq 60 \mathrm{~s}$ were searched for. We used both the wavelet and the Fourier analysis methods. The wavelet transform was used to analyze a non-stationary power (Daubechies 1990). The Morlet wavelet (Grossman \& Morlet 1984) representing a sinusoidal oscillation modulated by a Gaussian envelope was chosen as the analyzing wavelet. The wavelet parameter $\omega$ was set to 6 or 12 . As significance tests, the confidence levels at $80 \%$ and $95 \%$ are applied for the background spectrum modeled by the red and white noise, respectively. Regions where edge effects become

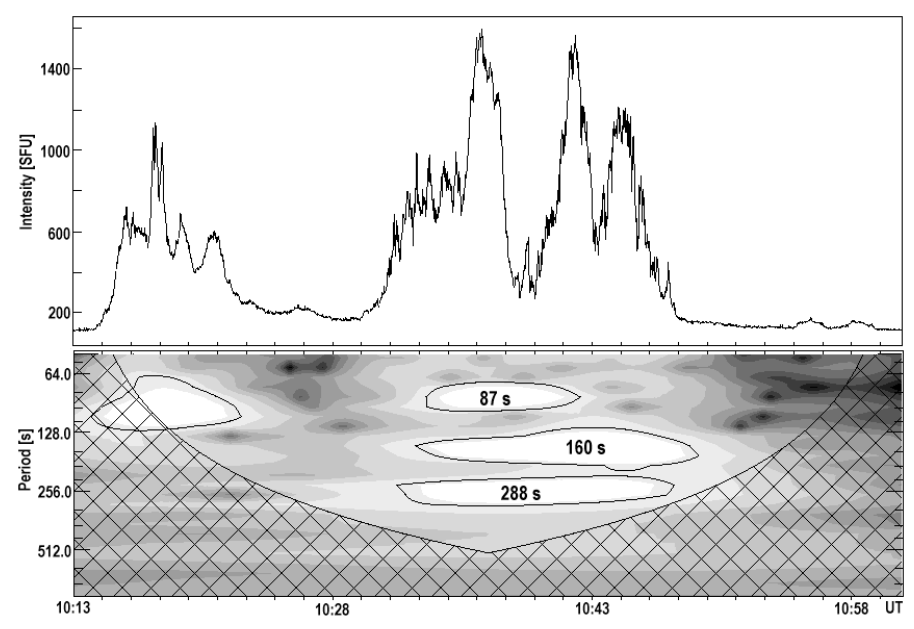

Fig. 7. The radio flux profile of the April 12, 2001 flare at $1.1 \mathrm{GHz}$ in the time interval 10:13-11:01 UT (top) and the wavelet power spectrum with characteristic periods 288,160 , and $87 \mathrm{~s}$ (white color areas) (bottom). The cross-hatched region is the COI.

important due to dealing with finite-length time series are labeled as the cone of influence (COI). The computation of all these parameters is performed as described by Torrence \& Compo (1998) using software based on the original routines of these authors ${ }^{1}$.

A typical example of the Morlet analysis made in this paper is shown in Fig. 7, where the $1.1 \mathrm{GHz}$ radio flux observed during the April 12, 2001 flare is analyzed. Among all more or less likely periods, we selected only the dominant ones. We consider only those fulfilling rather restrictive conditions: in the Fourier analysis those with the probability $\geq 75 \%$ (Mészárosová et al. 1999) and in the Morlet wavelet analysis those with significant

\footnotetext{
${ }^{1}$ http://paos.colorado.edu/research/wavelets/
} 
Table 4. Long periods ( $\geq 60$ s) found in the July 14, 2000, April 12, 2001, and April 15, 2001 flares in the HXT/YOHKOH channels L, M1, M2, $\mathrm{H}$, and the SXT/YOHKOH sources A, B, C, and D.

\begin{tabular}{cccc}
\hline $\begin{array}{c}\text { Yohkoh X-ray } \\
\text { channel } \\
\text { or source }\end{array}$ & $\begin{array}{c}\text { July 14, 2000 } \\
\text { Period } \\
{[\mathrm{s}]}\end{array}$ & $\begin{array}{c}\text { April 12, 2001 } \\
\text { Period } \\
{[\mathrm{s}]}\end{array}$ & $\begin{array}{c}\text { April 15, 2001 } \\
\text { Period } \\
{[\mathrm{s}]}\end{array}$ \\
\hline L & $116,76,61$ & 159,99 & \\
M1 & $330,315,147,78,60$ & 165,93 & $300,170,90,64$ \\
M2 & $330,315,147,79$ & $264,93,63$ & $300,111,77$ \\
H & $330,315,157,79$ & 264 & 98 \\
\hline A & & $320,140,60$ & \\
B & & 140 & \\
C & & 140,97 & \\
D & & 84 & \\
\hline
\end{tabular}

Table 5. Cross-correlation coefficients and time shifts between Ondřejov radio fluxes at some selected frequencies of the July 14 , 2000, April 12, 2001, and April 15, 2001 flares. Time shift values are present only for the cross-correlation coefficient $\geq 0.5$. The positive value of the time shift means that the radio flux at selected frequency remains behind that at $4.0 \mathrm{GHz}$. (CC means the cross-correlation)

\begin{tabular}{|c|c|c|c|c|c|c|c|c|}
\hline \multirow[b]{3}{*}{$\begin{array}{c}\text { Compared fluxes } \\
\text { at frequencies } \\
{[\mathrm{GHz}]}\end{array}$} & \multirow{2}{*}{\multicolumn{2}{|c|}{ "July 14,2000}} & \multirow{2}{*}{\multicolumn{2}{|c|}{ April 12, 2001}} & \multicolumn{4}{|c|}{ April 15, 2001} \\
\hline & & & & & \multicolumn{2}{|c|}{ 13:31:00-13:59:50 UT } & \multicolumn{2}{|c|}{$13: 59: 50-14: 11: 00 \mathrm{UT}$} \\
\hline & $\begin{array}{c}\mathrm{CC} \\
\text { coefficient }\end{array}$ & $\begin{array}{c}\text { Time } \\
\text { shift } \\
{[\mathrm{s}]}\end{array}$ & $\begin{array}{c}\mathrm{CC} \\
\text { coefficient }\end{array}$ & $\begin{array}{l}\text { Time } \\
\text { shift } \\
{[\mathrm{s}]}\end{array}$ & $\begin{array}{c}\mathrm{CC} \\
\text { coefficient }\end{array}$ & $\begin{array}{l}\text { Time } \\
\text { shift } \\
{[\mathrm{s}]}\end{array}$ & $\begin{array}{c}\mathrm{CC} \\
\text { coefficient }\end{array}$ & $\begin{array}{l}\text { Time } \\
\text { shift } \\
{[\mathrm{s}]}\end{array}$ \\
\hline $4.0 \& 0.81$ & - & - & - & - & 0.85 & 65 & 0.94 & 0 \\
\hline $4.0 \& 0.90$ & - & - & - & - & 0.85 & 65 & 0.94 & 0 \\
\hline $4.0 \& 1.00$ & - & - & - & - & 0.85 & 65 & 0.94 & 0 \\
\hline $4.0 \& 1.02$ & - & - & 0.02 & - & 0.85 & 65 & 0.85 & 0 \\
\hline $4.0 \& 1.10$ & - & - & 0.04 & - & 0.86 & 60 & 0.93 & 0 \\
\hline $4.0 \& 1.20$ & 0.42 & - & 0.23 & - & 0.85 & 53 & 0.77 & 0 \\
\hline $4.0 \& 1.30$ & 0.52 & 0 & 0.35 & - & 0.84 & 50 & 0.75 & 0 \\
\hline $4.0 \& 1.40$ & 0.65 & 0 & 0.49 & - & 0.82 & 45 & 0.74 & 0 \\
\hline $4.0 \& 1.50$ & 0.81 & 0 & 0.62 & -65 & 0.85 & 40 & 0.78 & 0 \\
\hline $4.0 \& 1.60$ & 0.94 & 0 & 0.70 & -60 & 0.85 & 35 & 0.78 & 0 \\
\hline $4.0 \& 1.70$ & 0.95 & 0 & 0.75 & -60 & 0.87 & 30 & 0.80 & 0 \\
\hline $4.0 \& 1.80$ & 0.96 & 0 & 0.79 & -50 & 0.89 & 30 & 0.76 & 0 \\
\hline $4.0 \& 1.90$ & 0.96 & 0 & 0.80 & -38 & 0.92 & 25 & 0.64 & 0 \\
\hline $4.0 \& 2.25$ & 0.97 & 0 & 0.83 & -10 & 0.96 & 20 & 0.63 & 0 \\
\hline $4.0 \& 2.50$ & 0.98 & 0 & 0.87 & -10 & 0.98 & 12 & 0.87 & 0 \\
\hline $4.0 \& 2.75$ & 0.98 & 0 & 0.95 & 0 & 0.98 & 10 & 0.96 & 0 \\
\hline $4.0 \& 3.00$ & 0.99 & 0 & 0.97 & 0 & 0.98 & 5 & 0.97 & 0 \\
\hline $4.0 \& 3.25$ & 0.99 & 0 & 0.98 & 0 & 0.99 & 3 & 0.99 & 0 \\
\hline $4.0 \& 3.50$ & 0.99 & 0 & 0.99 & 0 & 0.99 & 0 & 0.99 & 0 \\
\hline $4.0 \& 3.75$ & 1.00 & 0 & 1.00 & 0 & 0.99 & 0 & 0.99 & 0 \\
\hline
\end{tabular}

periods aside from the COI (see Fig. 7) and with respect to the white (95\% significant level) as well as to the red noise $(80 \%$ significant level). The periods that fulfilled these criteria are summarized in Table 3. In all studied flares we found periods in the interval of $60-513 \mathrm{~s}$. The periods can be divided into several groups: $\sim 300, \sim 150$, and $\sim 80 \mathrm{~s}$. There is no distinct difference in the periods found at low and high frequencies.

\subsection{Global analysis: Long period variations $\geq 60 \mathrm{~s}$ of Yohkoh hard and soft $X$-ray fluxes}

Using the same methods as in the previous section, we searched for the characteristic periods $\geq 60 \mathrm{~s}$ also in time series of the $\mathrm{X}$-ray fluxes. The results are summarized in Table 4 . The found periods are similar to those found in the radio emissions. It is interesting that one of the most distinct period ( $300 \mathrm{~s})$ was found in the April 12, 2001 flare in the soft X-ray ( 2 keV) emission, but not in hard X-rays. On the other hand this period was found in two other flares (July 14, 2001 and April 15, 2001).

\subsection{Cross-correlations of radio fluxes on different frequencies}

To understand the global relationship between radio fluxes on low and high frequencies (in the $0.8-4.5 \mathrm{GHz}$ range in our case) and to recognize different emission mechanisms as mentioned above, we made the cross-correlation of the radio fluxes at different frequencies during the whole July 14, 2000 and April 12, 2001 flares and in two distinct parts of the April 15, 2001 flare. The results are summarized in Table 5. In the case of the July 14, 2000 flare we can see that the crosscorrelation coefficient is very high if computed in the frequency interval 1.6-4.0 GHz. Below this frequency the relationship to the $4.0 \mathrm{GHz}$ flux is decreasing, which indicates a change of the emission mechanism (see also the radio spectrum in Fig. 1). The time shift is zero in this case. In the April 12, 2001 flare, for high frequencies $(\geq 2.5-4.0 \mathrm{GHz})$, we can see a similar relationship as in the July 14, 2000 flare. But, in the $1.5-2.5 \mathrm{GHz}$ frequency range, the cross-correlation coefficients remain relatively high, while the time shift shows a global positive frequency drift (see also the burst at the beginning of this flare and 

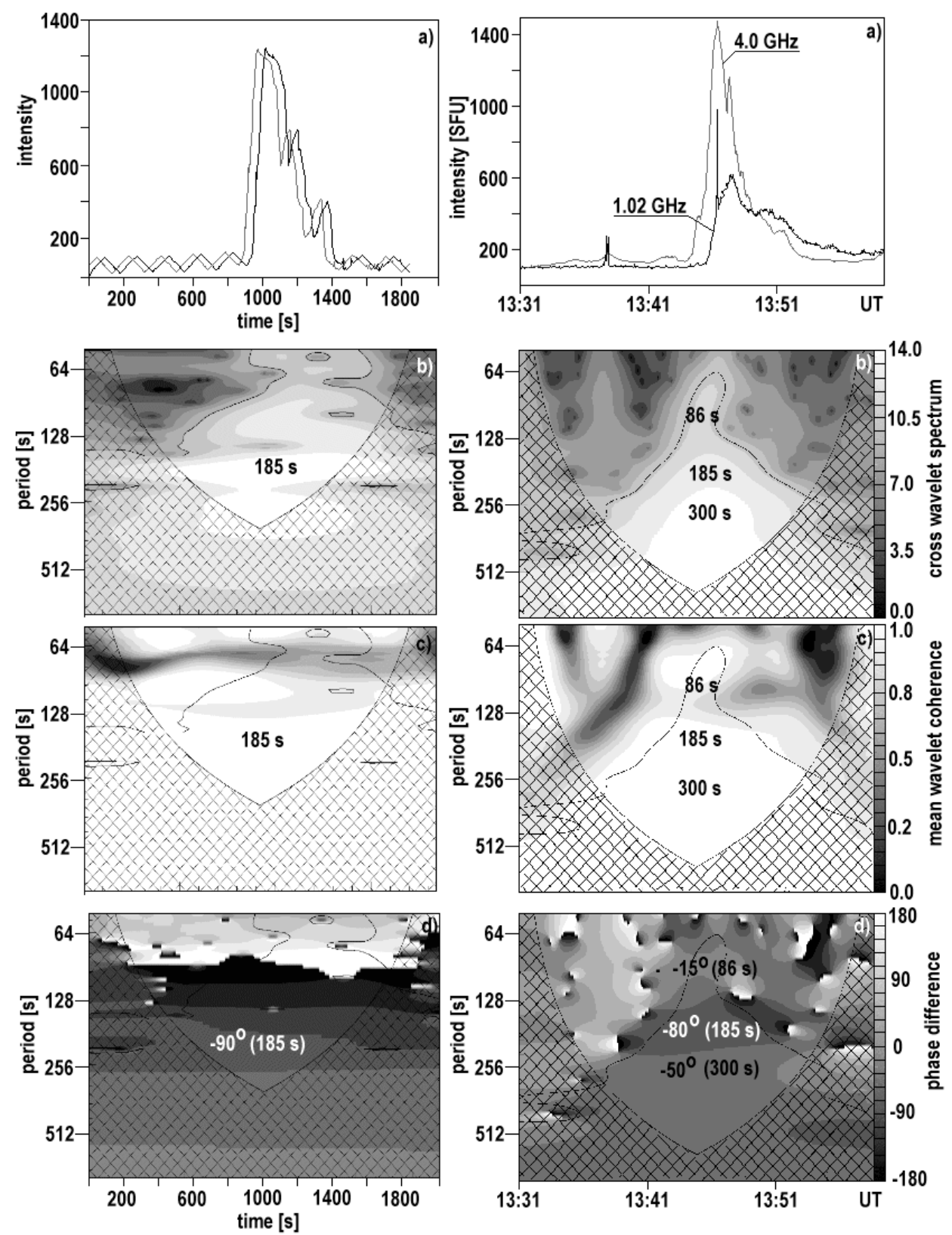

Fig. 8. Time data series a), their cross wavelet spectrum b), mean wavelet coherence c), and phase difference d) of an artificial test data and the April 15, 2001 drifting burst. Left panels: the test data series (in black and grey) are the same with a dominant period $185 \mathrm{~s}$ and phase difference $90^{\circ}$. The most significant range in the cross wavelet spectrum and the mean wavelet coherence are in white. Right panels: the same of the April 15, 2001 burst on frequencies 1.02 (in black) and $4.0 \mathrm{GHz}$ (in grey) during the time interval 13:31:00-13:59:50 UT, where the phase differences are $-15^{\circ}$, $-80^{\circ}$, and $-50^{\circ}$ for the dominant periods 86,185 , and $300 \mathrm{~s}$, respectively. Cross-hatched regions are the COIs. Absolute white corresponds to $180^{\circ}$ and absolute black to $-180^{\circ}$ (panels d)), while the contours overlaid are the same $95 \%$ confidence level (panels b) -d)). Gray bars on the right side of this figure are common for panels in both left and right columns.

its positive frequency drift (Fig. 3)). Analyzing the first part of the April 15, 2001 flare, we found high cross-correlations in the whole frequency range and the time shift expressing a clear frequency drift towards the low frequencies. On the other hand the second part of the April 15, 2001 burst is also highly correlated, but without time shift.

\subsection{Phase difference of periods between X-ray and dm-radio time series}

We used the method by Bloomfield et al. (2004) regarding the cross-wavelet spectrum, mean wavelet coherence, and phase differences of dominant periods between two individual time series.
This method utilizes the inherent phase information residing within the complex Morlet transform. We choose the complex Morlet wavelet (Torrence \& Compo 1998) as it yields a complex wavelet transform, containing information on both amplitude and phase as well as for its adequate shape relative to the studied variations. To understand the response of the wavelet coherence to noise, we randomized both time series adding reasonable random noise and calculated the coherence over 1000 separate realizations. While a wavelet power spectrum depicts the variance of a time series, with times of large variance showing large power, the cross-wavelet power of two time series depicts the covariance between these time series. This allows cross-wavelet power to be used as a quantified indication of the similarity of 
Table 6. Cross-wavelet spectrum, mean wavelet coherence, and phase difference for the periods in the Yohkoh X-ray and Ondřejov radio fluxes at selected frequencies of the April 12, 2001 and April 15, 2001 flares. The cross-wavelet spectrum values 11 and 0.0 mean the highest and lowest cross-correlations, respectively. The mean wavelet coherence values 1.0 and 0.0 mean the highest and lowest coherence levels, respectively. Range of the phase difference values is $-180^{\circ} \leq \Phi \leq 180^{\circ}$.

\begin{tabular}{ccccc}
\hline \hline $\begin{array}{c}\text { Compared X-ray \& radio } \\
\text { time series } \\
\text { channel \& frequency [GHz] }\end{array}$ & $\begin{array}{c}\text { Period } \\
{[\mathrm{s}]}\end{array}$ & $\begin{array}{c}\text { Cross-wavelet } \\
\text { value }\end{array}$ & $\begin{array}{c}\text { Coherence } \\
\text { value }\end{array}$ & $\begin{array}{c}\text { Phase } \\
\text { difference } \\
\text { [degree] }\end{array}$ \\
\hline L \& 1.20 & $90-100$ & 11.0 & 0.8 & -17 \\
L \& 1.20 & $155-165$ & 11.0 & $0.3-0.7$ & $-50--80$ \\
M1 \& 1.20 & 93 & 11.0 & 0.9 & -144 \\
M1 \& 1.20 & $160-170$ & 11.0 & $0.4-0.9$ & $-108--144$ \\
M2 \& 2.75 & $93-94$ & 9.2 & 0.7 & -18 \\
M2 \& 2.75 & $260-300$ & 10.0 & 1.0 & -50 \\
H \& 2.75 & $260-300$ & 9.0 & 1.0 & -18 \\
\hline M1 \& 1.20 & $60-70$ & $9.0-11.0$ & $0.4-0.7$ & -110 \\
M1 \& 1.20 & $170-190$ & 11.0 & $0.9-1.0$ & $-18--50$ \\
M1 \& 1.20 & 300 & 9.8 & $0.8-0.9$ & 18 \\
M2 \& 1.20 & 300 & 10.0 & 0.7 & $50-80$ \\
H \& 0.81 & $95-105$ & 10.0 & $0.7-0.2$ & 180 \\
\hline
\end{tabular}

power between two time series. Measurements of the phase difference between two time series yield information on the phase delay between oscillations in the time series as a function of periods.

To test the method, we applied it to two artificial data series (left panel a, Fig. 8), which are identical, but the second one (gray line) is shifted by $90^{\circ}$ compared to the first one (black line). They have the same period of $185 \mathrm{~s}$ as the basic part of the time series. Additionally, the time series contain an aperiodic variable added to the signal in an attempt to simulate the onset of the flare (right panel a, Fig. 8). The cross wavelet spectrum and the mean wavelet coherence of these artificial time series (left panels in Fig. 8) show us the most significant region (in white) for the dominant $185 \mathrm{~s}$ period. The phase difference (left plot d, Fig. 8) is about $90^{\circ}$ (in gray), which is in agreement with the shift originally introduced. Summarizing, we can conclude that this applied wavelet method, with its selected particular parameters, is adequate for applied analysis of the radio signals used in the paper.

This technique was applied to the selected radio and Yohkoh hard and soft X-ray fluxes of the July 14, 2000, April 12, 2001, and April 15, 2001 drifting bursts. Because of too low coherence between the radio and hard X-ray time series of the July 14, 2000 drifting burst, as well as between the radio and soft X-ray fluxes of the April 12, 2001 one, the phase differences cannot be established. The results are summarized in Table 6 for the hard X-ray (L, M1, M2, and H channels) and dm-radio fluxes of the April 12, 2001 and April 15, 2001 drifting bursts with similar dominant periods (see Tables 3 and 4 ).

The smaller phase differences $\left(<100^{\circ}\right)$ were found for rather higher periods ( $>200 \mathrm{~s}$, Table 6 ), for the Yohkoh hard X-ray L, $\mathrm{M} 2$, and $\mathrm{H}$ channels (April 12, 2001), and for the M1 and M2 channels (April 15, 2001). The higher phase differences $\left(>100^{\circ}\right)$ were found for rather smaller periods $(<200 \mathrm{~s})$, for the Yohkoh hard X-ray M1 channel (April 12, 2001), and for the M1 and H channels (April 15, 2001).

\subsection{Analysis of the unique drifting burst at the beginning of the April 15, 2001 flare}

At the beginning of the April 15, 2001 flare we found a rare broadband negatively slowly drifting burst. To understand its
Table 7. Cross-correlation coefficients and time shifts between radio and hard X-ray (M1, M2, H) fluxes observed during the drifting burst at the beginning the April 15, 2001 flare. The positive value of the time shift means that the radio flux at selected frequency stays behind the $\mathrm{X}$-ray flux.

\begin{tabular}{ccc}
\hline $\begin{array}{c}\text { X-ray channel } \\
\text { and radio frequency } \\
\text { [GHz] }\end{array}$ & $\begin{array}{c}\text { Cross-correlation } \\
\text { coefficient }\end{array}$ & $\begin{array}{c}\text { Time } \\
\text { shift } \\
{[\mathrm{s}]}\end{array}$ \\
\hline M1 \& 0.81 & 0.87 & 20 \\
M2 \& 0.81 & 0.80 & 5 \\
H \& 0.81 & 0.75 & 0 \\
M1 \& 4.00 & 0.84 & -65 \\
M2 \& 4.00 & 0.90 & -85 \\
H \& 4.00 & 0.85 & -10 \\
\hline
\end{tabular}

physical origin we study it in detail. First, the cross-correlations between radio and hard X-ray fluxes during this burst were analyzed. The results are summarized in Table 7 . It can be seen here that the hard X-ray emission is well correlated with the radio fluxes and that it is delayed several tens of seconds behind the $4.0 \mathrm{GHz}$ radio emission.

Then we applied the method by Bloomfield et al. (2004) to the drifting burst. Its time series at the frequencies 1.02 and 4.0 GHz, and their cross wavelet spectrum, mean wavelet coherence, and phase differences are in the right panels of Fig. 8. The best cross wavelet and coherence values are indicated. The phase differences are $-15^{\circ},-80^{\circ}$, and $-50^{\circ}$ for the dominant periods 86,185 , and $300 \mathrm{~s}$, respectively (right panel d).

This technique was applied to all of the selected radio fluxes of the April 15, 2001 drifting burst and the results are summarized in Table 8 . The highest cross wavelet and coherence values were found for the interval of significant periods 300-320 s. For lower values of significant periods the cross wavelet and coherence values decrease. The phase difference systematically increases towards lower frequencies, which corresponds to the increase of the time shift computed by the cross-correlation method in the previous paragraph. Furthermore the coherence values decrease in the interval of $1.3-1.7 \mathrm{GHz}$. The cause of this decrease can be found in the detailed view on the drifting burst (Fig. 9). Namely, in this frequency range an additional drifting burst appeared after 13:49 UT. 
Table 8. Cross-wavelet spectrum, mean wavelet coherence, and phase difference for the periods in the Ondřejov radio fluxes at selected frequencies of the April 15, 2001 drifting burst at 13:31:00-13:59:50 UT. The cross-wavelet spectrum values 14 and 0.0 indicate the highest and lowest crosscorrelations, respectively. The mean wavelet coherence values 1.0 and 0.0 indicate the highest and lowest coherences, respectively. The range of the phase difference values is $-180^{\circ} \leq \Phi \leq 180^{\circ}$.

\begin{tabular}{cccccccccc}
\hline \hline $\begin{array}{c}\text { Compared fluxes } \\
\text { at frequencies } \\
\text { [GHz] }\end{array}$ & $\begin{array}{c}\text { Cross- } \\
\text { wavelet } \\
\text { value }\end{array}$ & $\begin{array}{c}\text { Cohe- } \\
\text { rence } \\
\text { value }\end{array}$ & $\begin{array}{c}\text { Phase } \\
\text { difference } \\
\text { [degree] }\end{array}$ & $\begin{array}{c}\text { Cross- } \\
\text { wavelet } \\
\text { value }\end{array}$ & $\begin{array}{c}\text { Cohe- } \\
\text { rence } \\
\text { value }\end{array}$ & $\begin{array}{c}\text { Phase } \\
\text { difference } \\
\text { [degree] }\end{array}$ & $\begin{array}{c}\text { Cross- } \\
\text { wavelet } \\
\text { value }\end{array}$ & $\begin{array}{c}\text { Cohe- } \\
\text { rence } \\
\text { value }\end{array}$ & $\begin{array}{c}\text { Phase } \\
\text { difference } \\
\text { [degree] }\end{array}$ \\
\hline Period P [s]: & & $70-90$ & & & $160-190$ & & & $300-320$ & \\
\hline $4.0 \& 0.81$ & 10 & 0.7 & -180 & $11-12$ & 0.9 & -110 & $12-14$ & 1.0 & -80 \\
4.0 \& 0.90 & - & - & - & $11-12$ & 0.9 & -110 & $12-14$ & 1.0 & -50 \\
$4.0 \& 1.00$ & 10 & 1.0 & $-15--50$ & $11-12$ & 1.0 & -80 & $12-14$ & 1.0 & -50 \\
$4.0 \& 1.02$ & 11 & 1.0 & -15 & $11-12$ & 1.0 & -80 & $12-14$ & 1.0 & -50 \\
$4.0 \& 1.10$ & 10 & $0.9-0.8$ & +15 & $11-12$ & 1.0 & -80 & $12-14$ & 1.0 & -50 \\
$4.0 \& 1.20$ & - & - & - & $11-12$ & 1.0 & -80 & $12-14$ & 1.0 & -50 \\
$4.0 \& 1.30$ & 10 & $0.6-0.7$ & -50 & $11-12$ & $0.7-0.9$ & -50 & $12-14$ & $0.7-1.0$ & -50 \\
$4.0 \& 1.40$ & 11 & $0.4-0.7$ & -50 & $11-12$ & $0.6-0.8$ & -50 & $12-14$ & $0.2-0.9$ & -50 \\
$4.0 \& 1.50$ & 11 & $0.4-0.7$ & -50 & $11-12$ & $0.6-0.8$ & $-15--50$ & $12-14$ & $0.2-0.9$ & -15 \\
$4.0 \& 1.60$ & 11 & $0.5-0.8$ & -50 & $11-12$ & $0.6-0.8$ & $-15--50$ & $12-14$ & $0.2-0.9$ & -15 \\
$4.0 \& 1.70$ & 11 & $0.5-0.8$ & -15 & $11-12$ & $0.6-0.9$ & $-15--50$ & $12-14$ & $0.7-1.0$ & -15 \\
$4.0 \& 1.80$ & 11 & $0.5-0.8$ & $-15--50$ & $11-12$ & $0.8-1.0$ & $-15--50$ & $12-14$ & 1.0 & -15 \\
$4.0 \& 1.90$ & 11 & $0.7-0.9$ & -50 & $11-12$ & 1.0 & -15 & $12-14$ & 1.0 & -15 \\
$4.0 \& 2.25$ & 11 & 1.0 & -15 & $11-12$ & 1.0 & -15 & $12-14$ & 1.0 & -15 \\
$4.0 \& 2.50$ & 11 & 1.0 & $-15--50$ & $11-12$ & 1.0 & -15 & $12-14$ & 1.0 & -15 \\
$4.0 \& 2.75$ & 11 & 1.0 & -15 & $11-12$ & 1.0 & -15 & $12-14$ & 1.0 & -15 \\
$4.0 \& 3.00$ & 11 & 1.0 & -15 & $11-12$ & 1.0 & -15 & $12-14$ & 1.0 & -15 \\
$4.0 \& 3.25$ & 11 & 1.0 & -15 & $11-12$ & 1.0 & -15 & $12-14$ & 1.0 & -15 \\
$4.0 \& 3.50$ & 11 & 1.0 & -15 & $11-12$ & 1.0 & -15 & $12-14$ & 1.0 & -15 \\
$4.0 \& 3.75$ & 11 & 1.0 & 0 & $11-12$ & 1.0 & 0 & $12-14$ & 1.0 & 0 \\
\hline
\end{tabular}

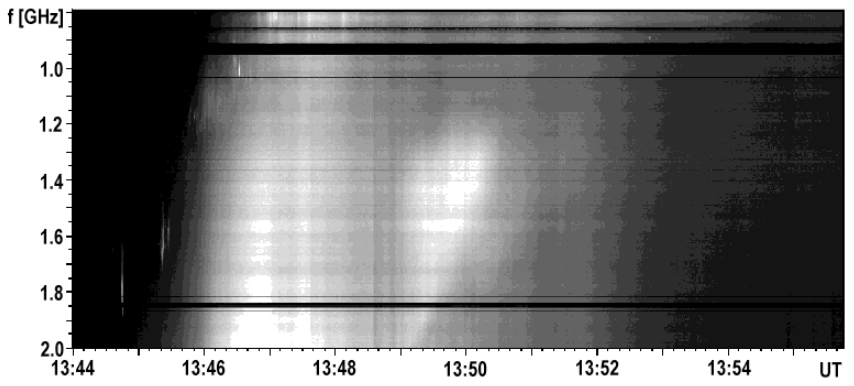

Fig. 9. Detail of the $0.8-2.0 \mathrm{GHz}$ radio spectrum of the broadband drifting burst observed at the beginning of the April 15, 2001 flare.

\section{Discussion and conclusions}

In the present statistical analysis of three X-class flares we found the characteristic periods in the interval of $60-513 \mathrm{~s}$ in the radio $(0.8-4.5 \mathrm{GHz})$ and $60-330 \mathrm{~s}$ in the X-ray Yohkoh fluxes. The cross-correlation between radio fluxes at different frequencies for the July 14, 2000 and April 12, 2001 flares confirmed that the radio spectra in the $0.8-4.5 \mathrm{GHz}$ range consist of two bands of radio emissions. Due to the results of the cross-correlations and because of fine structures on lower frequencies, we suppose that the broadband pulsating emission below $\sim 1.4 \mathrm{GHz}$ is generated by the plasma emission mechanism, and the broadband emission in the range above $\sim 1.4 \mathrm{GHz}$, which is smooth and without fine structures, by the gyro-synchrotron emission mechanism. However, the characteristic periods in both the radio bands are similar.

It is interesting that the period of about $300 \mathrm{~s}$ was found not only in variations of the radio flux in the whole $0.8-4.5 \mathrm{GHz}$ range in three X-class flares, but also in the X-ray loop-top emission of the April 12, 2001 flare. Because these $300 \mathrm{~s}$ variations were found for both the gyro-synchrotron and plasma emissions at layers of the solar atmosphere where the plasma $\beta$ parameter is considered to be low, we think that these variations are due to variations of the magnetic field that modulate the flare reconnection process including the particle acceleration. This period corresponds to that of the well known 5-min photospheric oscillations. It can indicate a connection between flare and photospheric processes as proposed by Kislyakov et al. (2006). If it is the case, then the photosphere represents an oscillating boundary condition for the reconnection process in the low corona. Loops anchored in the photosphere are forced by the 5-min photospheric oscillations to interact, which leads to the 5-min modulation of the flare processes. It is important to mention that the same $300 \mathrm{~s}$ period was also observed in the "quiet" solar atmosphere above the boundary of supergranular cells (Gömöry et al. 2006).

There is an alternative explanation that was proposed by Nakariakov et al. (2006). In the flare region or in its close vicinity there is a loop, which due to the flare or other perturbations, starts to oscillate with the $300 \mathrm{~s}$ period. This oscillating loop produces in its vicinity the MHD waves that penetrate into the flare current sheet and modulate the electric resistivity, thus causing the reconnection process to vary with the $300 \mathrm{~s}$ period. In this model the $300 \mathrm{~s}$ period is given by the physical parameters of the oscillating loop, and there is no direct connection to the photospheric oscillations. Nevertheless, to distinguish between these two explanations of the $300 \mathrm{~s}$ period, further detailed studies of the flare oscillations are necessary.

It was found that the April 15, 2001 flare in the $0.8-4.5 \mathrm{GHz}$ range started with a unique broadband slowly negatively drifting burst in association with the flare loop ejection observed by TRACE. The detailed analysis of this burst shows that it consists of two parts. The cross-correlation and coherence methods revealed that not only the burst as a whole is drifting, but also all features (substructures) in the main part of the burst drift simultaneously towards lower frequencies. Such results cannot be explained in the model based on the gyro-synchrotron emission 
mechanism. Namely, in the gyro-synchrotron model the substructures should be synchronized in time. Therefore, the main part of the drifting structure appear to be generated by plasma emission processes. On the other hand, the additional part of this burst (which disturbed the coherence) could be generated by the gyro-synchrotron mechanism.

Acknowledgements. We thank Dr. V. M. Nakariakov for his useful remarks that improved this paper. H.M., M.K., F.F., and K.J. acknowledge support from the project AV0Z10030501 and the grant IAA3003202 from the Academy of Sciences of the Czech Republic; J.R. acknowledges support from the VEGA grant 02/6195/26 from the Slovak Academy of Sciences. This research is part of the European Solar Magnetism Network (EC/RTN contract HPRN-CT-2002-00313). H.M. wishes to express her gratitude for the kind hospitality of and fruitful discussions with Prof. A. V. Stepanov and Prof. A. A. Soloviev from the Central Astronomical Observatory at Pulkovo in St. Petersburg, Russia. The wavelet analysis was performed using the software based on tools provided by C. Torrence and G.P. Compo at http: //paos. colorado. edu/research/wavelets/.

\section{References}

Aschwanden, M. J. 2005, MHD Oscillations, in Physics of the Solar Corona (Chichester, UK: Praxis Publishing) 283

Aschwanden, M. J., Fletcher, L., Schrijver, C. J., \& Alexander, D. 1999, ApJ, 520,880

Aschwanden, M. J., De Pontieu, B., Schrijver, C. J., \& Title, A. M. 2002, Sol. Phys., 206, 99
Aurass, H., \& Mann, G. 1987, Sol. Phys., 112, 359

Bloomfield, D. S., McAteer, R. T. J., Lites, B. W., et al. 2004, ApJ, 617, 623

Chernov, G. P., Merkeev, A. K., Poquerusse, M., et al. 1998, A\&A, 334, 314

Daubechies, I. 1990, IEEE Trans. Inform. Theory, 36, 961

De Moortel, I., Ireland, J., \& Walsh, R. W. 2000, A\&A, 355, L23

Fleishman, G. D., Stepanov, A. V., \& Yurovsky, Yu. F. 1994, Sol. Phys. 153, 403

Gelfreikh, G. B., Grechnev, V., Kosugi, T., \& Shibasaki, K. 1999, Sol. Phys., 185,177

Grossman, A., \& Morlet, J. 1984, SIAM J. Math. Anal., 15, 723

Gömöry, P., Rybák, J., Kučera, A., Curdt, W., \& Wöhl, H. 2006, A\&A, 448, 1169 Jiřička, K., Karlický, M., Kepka, O., \& Tlamicha, A. 1993, Sol. Phys., 147, 203

King, D. B., Nakariakov, V. M., Deluca, E. E., Golub, L., \& McClements, K. G. 2003, A\&A, 404, L1

Kislyakov, A. G., Zaitsev, V. V., Stepanov, A. V., \& Urpo, S. 2006, Sol. Phys., 233, 89

Kliem, B., Karlický, M., \& Benz, A. O. 2000, A\&A, 360, 715

Mészárosová, H., Jiřička, K., \& Karlický, M. 1999, A\&A, 348, 1005

Nakariakov, V. M., \& Ofman, L. 2001, A\&A, 372, L53

Nakariakov, V. M., \& Verwichte, E. 2005, Coronal Waves and Oscillations, Living Rev. Sol. Phys., 2, 3; Online Article: cited Sept. 2005; http: //www . livingreviews.org/lrsp-2005-3

Nakariakov, V. M., Tsiklari, D., Kelly, A., Arber, T. D., \& Aschwanden, M. J. 2004, A\&A, 414, L25

Nakariakov, V. M., Foullon, C., Verwichte, E., \& Young, N. P. 2006, A\&A, 452, 343

Nindos, A., Alissandrakis, C. E., Gelfreikh, G. B., Bogod, V. M., \& Gontikakis, C. 2002, A\&A, 386, 658

Ogawara, Y., Takano, T., Kato, T., \& Kosugi, T., et al. 1991, Sol. Phys., 136, 10 Torrence, C., \& Compo, G. P. 1998, Bull. Am. Meteorol. Soc., 79, 61 